\title{
IMPACTO DEL AMBIENTE EN LA EXPRESIÓN VOLITIVA DE ADULTOS MAYORES QUE PARTICIPAN EN TALLER DE MÚSICA
}

\author{
ENVIRONMENTAL IMPACT ON THE VOLITIVE EXPRESSION OF ELDERLY PEOPLE \\ WHO PARTICIPATES IN THE NURSING HOME'S MUSIC WORKSHOP
}

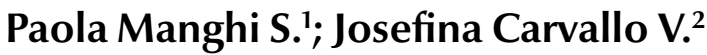

\begin{abstract}
RESUMEN
El presente estudio tiene como objetivo describir el impacto del ambiente en la expresión de la volición de los adultos mayores con demencia leve a moderada, que participan en taller de música de una residencia en Santiago. Para ello fue necesaria la realización de una investigación de tipo cualitativa descriptiva, donde se llevó a cabo la observación del taller de música durante 1 mes (4 sesiones), utilizando el instrumento de evaluación "Cuestionario Volicional" a cuatro integrantes. Los principales resultados corresponden al impacto que provoca el ambiente (físico y social) sobre la expresión volitiva, según las tareas o formas ocupacionales observadas en cada sesión. Desde el ambiente físico los participantes alcanzan una expresión volitiva espontánea al momento de cantary/o tocar instrumentos relacionados a sus intereses, como por ejemplo canciones judías y de su época. A su vez, el ambiente social interfiere en la expresión volitiva debido a diferentes factores relacionados con la forma ocupacional del taller, restringiendo la participación de los integrantes. El siguiente desafío está en profundizar, en futuras investigaciones, en cómo impacta el ambiente en su participación ocupacional en la expresión volitiva en adultos mayores con demencia.
\end{abstract}

\section{PALABRAS CLAVES}

Adulto mayor, impacto ambiental, volición, demencia, música.

1 Terapeuta Ocupacional, licenciada en ocupación humana, Universidad Mayor. Diplomado en Neuropsicología y Neuropsiquiatría del adulto, Universidad Católica. Diplomado en Investigación en Medicina Basada en la Evidencia, Universidad de los Andes. Diplomado en Psicogerontología Social y Educativa, Universidad Católica. Diplomado en Modelo de Ocupación Humana, Universidad de los Andes. ResearchedID: B-9756-2017. ORCID: B-9756-2017. Correo postal: 8420584. Email: pamnghi@miuandes.cl № teléfono: +56 999152572

2 Terapeuta Ocupacional, Licenciada en Ciencias de la Ocupación Humana, Universidad de los Andes. Instituto de afiliación principal: Universidad de los Andes. ResearchedID: J-3100-2017. ORCID: 0000-0002-0930-5500. Correo postal: 7590943. Email: jcarvallo@miuandes. cl. 


\section{ABSTRACT}

The objective of the present study is to describe the environment impact over the volition expression of elderly people with low to moderate dementia, who participate on a music workshop in a Nursing Home. A qualitative-descriptive type investigation was done, developed by the observation of the workshop for one month (four sessions), using the "Volitional Questionnaire" evaluation instrument on four of all the participants that participate in the workshop. The main results involved the impact of the environment over the expression of the volition of the group, according to the task or occupational forms observed on each session, where the participants reached a spontaneous volition expression when they are singing or playing instruments related to their interests, like jewish or classic songs. Furthermore, the research shows that the social environment, related to the occupational forms, also generates an impact on the volition expression, which is observed as other types of difficulty for that expression, with also the difficulty for the group on the participation on the workshop.

Finally, the main conclusions are the need to deepen the research related to the elderly dementia's volitive component, and how the environment impacts their occupational participation.

\section{KEYWORDS}

Aged, environmental, volition, dementia, music

Recibido: 15/05/2017

Aceptado: 17/10/2017 


\section{INTRODUCCIÓN}

El presente estudio pretende describir el impacto que provoca el ambiente físico y social sobre el componente volitivo de adultos mayores con demencia leve a moderada, con el fin de profundizar en torno a las formas ocupacionales o tareas. Dentro de este estudio se espera encontrar cómo impacta el ambiente en la expresión volitiva, relacionadas a las diferentes tareas o formas ocupacionales observadas durante el taller, así como también se espera que el ambiente, físico, socialy cultural emplee un rol sobre dicha expresión. El contexto de participación en el cual se desarrolla esta investigación es un taller de música y el principal sustento teórico está dado por el Modelo de Ocupación Humana.

Con esta investigación se busca brindar evidencia de cómo el ambiente facilita o restringe la participación ocupacional de los adultos mayores en una residencia y poder aportar a mejorar las intervenciones y acercamientos desde el área de la salud desde la implementación de talleres en otros centros de atención del adulto mayor en el país.

\section{EL ENVEJECIMIENTO EN CHILE}

Durante las últimas décadas Chile ha sido testigo del crecimiento de la población de adulto mayor (persona de 60 años o más) por sobre el resto de la población, debido a un aumento en la esperanza de vida y disminución en la tasa de natalidad, representando así a un $15,6 \%$ de la población total. Dicha cifra se encuentra en aumento, proyectando que para el año 2025 las personas mayores tendra porcentaje mayor que la población de personas menores de 15 años (Baranda, 2014). Asimismo, se ha observado un incremento en la prevalencia de enfermedades crónicas dentro de dicha población, acrecentando el riesgo y niveles de vulnerabilidad, fragilidad, limitaciones funcionales y finalmente de dependencia en el adulto mayor (SENAMA, 2013). Dentro de este mismo marco cabe mencionar el incremento de la prevalencia de demencias en la población mayor, constituyendo un factor determinante de dependencia e institucionalización en dicha comunidad.

El proceso de envejecimiento lleva consigo diversos cambios, tanto a nivel físico, psicológico, emocional, social, entre otros. Existe, además, un enlentecimiento en las funciones fisiológicas, lo que puede llevar a un deterioro en esta índole, incrementando las probabi- lidades de generar un estado de fragilidad, llevando a una pérdida en la capacidad funcional, estado de vulnerabilidad y finalmente hacia la dependencia (Ayala, 2010). Desde la mirada de la Terapia Ocupacional (T.O), el deterioro progresivo en el adulto mayor podría llevar a la disfunción ocupacional. Esta podría verse afectada por la disminución de sus habilidades de desempeño, lo cual provocaría un desequilibrio entre las demandas del entorno y su desempeño ocupacional. Más aún, en adultos mayores con demencia, donde se puede observar creciente dificultad en la adaptación por motivos del deterioro orgánico, conductuales, emocionales y psicológicos, aspectos propios de la patología.

\section{DEMENCIA}

En la quinta versión de la Guía de Consulta de los Criterios Diagnósticos del DSM, la Demencia se encuentra como Trastorno Neurocognitivo, y éste es definido como: "Un declive cognitivo significativo comparado con el nivel previo de rendimiento en uno o más dominios cognitivos (atención compleja, función ejecutiva, aprendizaje y memoria, lenguaje, habilidad perceptual motora o cognición social)" (Asociación Americana de Psiquiatría, 2014, p 334).

En la demencia se describen diversos cambios que afectan a la vida ocupacional de la persona, a nivel inicial se observan dificultades relacionadas a la función ejecutiva, lo que se refleja en problemas para manejar, trabajary de seguridad en el hogar, ya que hay deterioro en la memoria, dificultad para resolver problemas y de orientación témporo-espacial, entre otras cosas; lo que puede comenzar a generar dificultades para el desempeño satisfactorio de la persona dentro de las actividades de su rutina diaria. En estados más avanzados, dichas dificultades aumentan, generando problemas para el desempeño en actividades básicas de la vida diaria, tales como auto-alimentación, movilidad, baño, aseo personal, entre otros (American Occupational Therapy Association [AОтA], 2015), lo que provoca mayor pérdida de la capacidad funcional de la persona, aumentando su nivel de dependencia y, por ende, disminuye su sentido personal de auto-valía, llevando a la disminución del interés por participar en ocupaciones y en otros contextos.

\section{MODELO DE OCUPACIÓN HUMANA}

El Modelo de Ocupación Humana (моно), el cual presenta la base teórica para el desarrollo de la presente 
investigación, corresponde a un modelo conceptual de la práctica de la terapia ocupacional, que "conceptualiza a las personas como compuestas por tres subsistemas interrelacionados: volición, habituación y capacidad de desempeño (...). Estos tres elementos, en interacción con el ambiente, definen las cosas que elegimos hacer, la manera en que organizamos nuestras vidas, y lo que somos capaces de hacer" (De las Heras, Geist, Kielhofner \& li, 1998, p. 4). Específicamente la investigación se enfocará en la volición de las personas, y el impacto ambiental observable durante la participación de los adultos mayores en un taller que es signficativo para ellos (taller de música).

El presente modelo, además de aportar con las bases teóricas para el proceso de investigación, posee instrumentos de evaluación. En este caso, se utilizó el Cuestionario Volicional VQ, el cual esto permitirá el posterior análisis y generación de resultados y conclusiones atingentes.

\section{VOLICIÓN, AMBIENTE Y DEMENCIA}

"La volición o motivación para participar en ocupaciones es, en el nivel más básico, una necesidad innata de las personas para actuar en el mundo. Todos los seres humanos se sienten atraídos por la acción. Eventualmente, la volición puede guiar acciones y elecciones. Así, la volición, que nos impulsa a actuar en el mundo, puede facilitar la autoorganización y guiar el autoconocimiento" (De las Heras, Llerena, Kielhofner, 2003, p 4). Así es como, en la etapa de la vida de la adultez mayor, nos encontramos con dinámicas que se entrelazan entre los recuerdos, la vida actual, lo que nos lleva a que su desempeño esté marcado por la interpretación de las experiencias. "En cualquier instante en el tiempo, la volición de la persona reflejará una historia y circunstancias personales únicas que la han formado y continúan formándola" (Kielhofner, 2011. P 35 ).

Para el adulto mayor con demencia este escenario es diferente, es como si experimentaran un constante aprendizaje, pues presentan dificultad para evocar el recuerdo espontáneo sobre vivencias y experiencias del presente, llevándolos a frustraciones y disminuciones en su volición general. Desde ahí, que para los profesionales del área de la salud y/o geriatría, es importante la aplicación de elementos facilitadores de dichos recuerdos, a través de la historia de vida y/o del apoyo familiar (red de apoyo más cercana que nos puede proporcionar información relevante de la persona). El profesional, al reconocer gustos e intereses pasados de la persona, puede presentárselos al adulto mayor, de modo que éstos recuerden en su subjetividad la participación en ellos y las experiencias vividas.

El моно define al entorno como las "características físicas y sociales particulares del contexto específico en el que uno hace algo, que impactan sobre lo que uno hace y cómo lo hace" (Kielhofner, 2011 p 98); asimismo, propone que el impacto ambiental se refiere a cómo influye éste en dar oportunidad, apoyo, demanda o restricción que los aspectos físicos y sociales (Kielhofner, 2011). Así como el entorno físico-social puede generar un impacto (positivo o negativo) sobre la persona y su participación en ocupaciones; la cultura, también influye en ésta y en las elecciones ocupacionales.

Dentro de la residencia existe una cultura religiosa predominante al Judaísmo. La mayoría de los residentes pertenecen a dicha religión y en sus vidas vivieron acorde a sus reglas y normas, así como también formaron parte de sus celebraciones y rituales. Específicamente en el taller de música, se respeta y promueve dicha cultura, lo cual facilita que el grupo de adultos mayores que asisten pueda participar de forma más efectiva.

\section{TALLER DE MÚSICA}

La decisión de realizar la investigación dentro del taller de música que ofrece la residencia radica en el hallazgo de algunos estudios que comentan sobre el impacto y beneficios que tiene la música sobre los adultos mayores con demencia. En el estudio de Jacobsen, Stelzer, Fritz, Chételat, La Joie y Turner (2015) se ha demostrado que la zona cerebral a cargo de la recepción y memoria musical se encuentra menos dañada en demencias de tipo Alzheimer en etapas moderadas a avanzadas, concluyendo que son de las últimas zonas cerebrales en dañarse por la atrofia o deterioro cerebral orgánico. A partir de esto, se entregan las bases neurológicas para la intervención de la persona con demencia desde el estímulo musical. Al promover la expresión de respuestas se podría facilitar un mayor grado de participación en la actividad, a partir de la evocación de recuerdos y memorias de sí mismo. Con ello, se logra desde la composición de la identidad, hasta facilitar la ejecución de alguna tarea o actividad guiada, estructurada o aprendida, dependiendo del caso. 


\section{MÉTOdo}

El desarrollo del presente estudio se hizo sobre la base de una investigación de tipo cualitativa descriptiva. Cabe destacar que previo a la ejecución de ésta, se requirió contar con la aprobación y permisos pertinentes tanto de la institución, como de los residentes seleccionados y sus familiares a cargo, a quienes se les solicitó firmar un consentimiento y asentimiento informado, en donde manifestaron su la voluntad por participar. A su vez, se solicitó la aprobación de la investigación al Comité de Ética de la Universidad de los Andes y al Servicio de Salud Metropolitano Oriente.

La población abordada corresponde al grupo de adultos mayores que participan de forma habitual en el taller de música. Para poder realizar la selección se basó fundamentalmente en los adultos mayores que presentaran un diagnóstico de demencia en estado leve a moderado, obtenido a través del diagnóstico médico y las pautas de evaluación cognitivas correspondientes: Mini Mental State Examination (MMSE) (Folstein, Folstein, McHugh \& Fanjiang, 1975) y Short Portable Mental Status Questionnaire de Pfeiffer (SPMSQ) (Pfeiffer, 1974). Y además que puedan expresaron la voluntad de participar en la investigación.
Por otro lado, como criterio de exclusión, los usuarios no podían presentar algún diagnóstico psiquiátrico, ni un mayor nivel de deterioro que impidiese la elección a conciencia y voluntaria de participar en el estudio. Por lo que finalmente la muestra se compone de cuatro residentes, de los cuales son tres mujeres y un hombre.

Para recoger la información se utilizó el "Cuestionario volicional (VQ)", desarrollado por Carmen Gloria de las Heras, Rebecca Geist, Gary Kielhofner y Yanling Li en el año 1998, basándose en el MOHO, el cual permite la evaluación de la volición de las personas a través de la observación de su participación en determinados ambientes, accediendo, además, a la obtención de información sobre el impacto de tal ambiente sobre la volición.

Para la utilización del instrumento y obtener la información atingente a la investigación fue necesaria la observación del taller de música en un período de un mes, observando un total de 4 sesiones. Posterior a ello se llevó a cabo el proceso de análisis de datos (TABLA 1), para finalmente generar las diferentes conclusiones y puntos de discusión del estudio.

TABLA 1 RESUMEN DE LA EVALUACIÓN DEL VQ, OBSERVACIÓN DURANTE 4 SESIONES EN TALLER DE MÚSICA

\begin{tabular}{|c|c|c|c|c|c|c|c|c|c|c|c|c|c|c|c|c|}
\hline \multirow{3}{*}{$\begin{array}{l}\text { ITEM } \\
\text { Muestra curiosidad }\end{array}$} & \multicolumn{16}{|c|}{ FECHA } \\
\hline & \multicolumn{4}{|c|}{$05 / 07$} & \multicolumn{4}{|c|}{$12 / 07$} & \multicolumn{4}{|c|}{ 19/07 } & \multicolumn{4}{|c|}{$02 / 08$} \\
\hline & $P$ & $\mathrm{D}$ & I & $\mathrm{E}$ & $\mathrm{P}$ & $\mathrm{D}$ & I & $\mathrm{E}$ & $\mathrm{P}$ & $\mathrm{D}$ & I & $\mathrm{E}$ & $P$ & $\mathrm{D}$ & I & $\mathrm{E}$ \\
\hline Inicia acciones/tareas & $P$ & $\mathrm{D}$ & I & $E$ & $\mathrm{P}$ & $\mathrm{D}$ & I & $\mathrm{E}$ & $\mathrm{P}$ & $\mathrm{D}$ & I & $\mathrm{E}$ & $P$ & $\mathrm{D}$ & I & $\mathrm{E}$ \\
\hline Intenta cosas nuevas & $P$ & $\mathrm{D}$ & I & $\mathrm{E}$ & $P$ & D & I & $\mathrm{E}$ & $\mathbf{P}$ & $\mathrm{D}$ & I & $E$ & $P$ & $\mathrm{D}$ & I & $\mathrm{E}$ \\
\hline Muestra preferencias & $P$ & $\mathrm{D}$ & I & $E$ & $\mathrm{P}$ & $\mathrm{D}$ & I & $\mathrm{E}$ & $\mathrm{P}$ & $\mathrm{D}$ & I & $E$ & $P$ & D & I & E \\
\hline $\begin{array}{l}\text { Muestra que una actividad es } \\
\text { especial o significativa }\end{array}$ & $P$ & $\mathrm{D}$ & I & $\mathrm{E}$ & $\mathrm{P}$ & $\mathrm{D}$ & I & $\mathrm{E}$ & $\mathrm{P}$ & $\mathrm{D}$ & I & $\mathrm{E}$ & $P$ & $\mathrm{D}$ & I & $\mathrm{E}$ \\
\hline Permanece involucrado & $\mathrm{P}$ & $\mathrm{D}$ & I & $E$ & $\mathrm{P}$ & $\mathrm{D}$ & I & $\mathrm{E}$ & $\mathrm{P}$ & $\mathrm{D}$ & I & $\mathrm{E}$ & $P$ & $\mathrm{D}$ & I & $\mathrm{E}$ \\
\hline Trata de corregir errores & $\mathrm{P}$ & $\mathrm{D}$ & I & $E$ & $\mathrm{P}$ & $\mathrm{D}$ & I & $\mathrm{E}$ & $P$ & $\mathrm{D}$ & I & $\mathrm{E}$ & $P$ & D & I & $E$ \\
\hline $\begin{array}{l}\text { Realiza una actividad hasta } \\
\text { completarla/lograrla }\end{array}$ & $P$ & $\mathrm{D}$ & I & $E$ & $P$ & D & I & $\mathrm{E}$ & $P$ & $\mathrm{D}$ & I & $\mathrm{E}$ & $P$ & D & I & $\mathrm{E}$ \\
\hline
\end{tabular}

PLOMO: observadas. NEGRO: no observadas.

CLAVE: $\mathbf{P}=$ Pasivo $/ \mathrm{D}=$ Dudoso $/ \mathrm{I}=$ Involucrado $/ \mathrm{E}=$ Espontáneo 


\section{Resultados}

Dentro de los resultados del presente estudio cabe destacar que el impacto del ambiente en el aumento de la expresión volitiva se observa durante las actividades del canto judío (al inicio y final) al utilizar instrumentos. En particular, cuatro de todos los ítems de evaluación del VQ son los que evidencian mayores cambios en la participante. Dichos ítems corresponderán a las categorías de análisis para la formulación de conclusiones, los cuales son:

- Muestra curiosidad.

- Inicia acciones y tareas.

- Permanece involucrado.

- Realiza una actividad hasta lograrla/completarla.

El ítem muestra curiosidad "evalúa si la persona explora objetos, eventos, tareas y personas en el ambiente. Se muestra tanto al orientar el cuerpo hacia o mirar, tocar y manipular objetos, como al hacer preguntas sobre los objetos, personas y tareas que el ambiente ofrece" (De las Heras et al, 1998, p.18). Éste se observa en la totalidad de la muestra, expresa variación de la volición a lo largo de la sesión; las formas ocupacionales impactan en dicha variación. Tres de los cuatro participantes demuestran curiosidad de forma espontánea, intentando recordar las canciones e inician cantos; en cambio, sólo uno, requiere de apoyo verbal y físico para notar la letra y comenzar a poner atención a la actividad, dicho apoyo debe mantenerse durante la tarea. El hecho de que demuestren curiosidad, a pesar del apoyo, implica la demostración de interés por la actividad, facilitando su participación y conexión durante la sesión.

Otra instancia, al tocar los instrumentos (objetos desde las formas ocupacionales), como sonajeros y maracas, de tamaño mediano, para ser maniobrados por el grupo observado, fáciles de utilizar, y conocidos para ellos, con lo que logran dar significado de diversión y ritmo a la actividad, presentando atributos de tipo sensorial, como texturas y emisión de sonidos. El conjunto de atributos sensoriales y significado relacionado a los objetivos, de cada instrumento, facilita el despertar de la curiosidad de la muestra, observándose más atentos y conectados, permitiendo la interacción entre ellos.

El ítem de Inicia Acciones y Tareas corresponde a la motivación intrínseca suficiente y la curiosidad por una actividad tal, que la persona es capaz de iniciar cualquier tipo de acción por dicha tarea, es decir, comienza a involucrarse en ésta y a realizar acciones relacionadas, las cuales por lo general surgen desde la espontaneidad de la propia persona.

En diversas ocasiones del taller, se observa al grupo con un nivel de curiosidad e involucramiento bajo, se aprecian algunos aspectos desde la forma ocupacional del taller, que limitan o dificultan tales acciones. La complejidad de la tarea, en algunas ocasiones se encontró por sobre las posibilidades de participación de los residentes, por ejemplo, las canciones utilizadas fueron en un idioma no dominado por los participantes, así como también se realizan actividades que consisten en la descripción verbal, sin apoyos, perdiendo la atención en la tarea. Dichas actividades se encontraron por sobre las habilidades y capacidades de los residentes, restringiendo su participación y observándose una limitación para la expresión de su volición, en torno a elementos como la exploración, determinación de objetivos y metas personales, e intentar cosas nuevas como la búsqueda de desafíos o responsabilidades adicionales, entre otros.

Raber, Teitelmam, Watts y Kielhofner (2010), en un estudio fenomenológico sobre la volición de los adultos mayores con demencia moderada a severa en sus AVD, describen que existe una falta en la iniciación espontánea de las ocupaciones o actividades por parte de los adultos mayores con deterioro cognitivo, por lo cual en diversas ocasiones era necesario adaptar las ocupaciones para favorecer la participación espontánea. Ello se correlaciona con lo observado en el taller de música, ya que, tal como fue descrito, dichas actividades no fueron modificadas o adaptadas para el beneficio de la participación de los usuarios, disminuyendo su expresión de la volición y su participación en éstas.

Otro ítem es el de Permanecer Involucrado, el cual corresponde al grado de conexión física y emocional que presenta la persona durante su desempeño en una tarea determinada, por lo que para poder observarlo se deben evidenciar cambios en la expresión facial, corporal o gestual durante la actividad (De las Heras et al, 1998). Se considera que una fracción de la participación en una actividad motivante es el factor emocional que sostiene a la persona para sentir el interés, la curiosidad y la intención de participar y mantenerse participando de dicha actividad o tarea.

Durante las diferentes sesiones se perciben diversas situaciones que facilitan la conexión emocional con la actividad, como son los cánticos y melodías judías, la 
recreación de situaciones familiares o conocidas, junto con evocar recuerdos o emociones; como ocurrió en una actividad donde los residentes debían arrojar a una bolsa, de forma gestual o simbólico-representativo, pensamientos y/o sentimientos negativos que presentaran en ese momento y en otra bolsa echar sueños y deseos. Esa simple actividad facilitó que los residentes buscaran y evaluaran cada situación y sensación como algo positivo o negativo, evaluando si se podía echar a la bolsa. Ello permitió que cada uno se vinculara con su propia historia, junto con el resto del grupo durante esa sesión.

El ambiente social también genera un impacto negativo en la participación de los adultos mayores en el taller. Asimismo se detalla en la investigación de Teitelman, Raber Wats (2010), sobre la fuerza del ambiente social dentro de la motivación y el compromiso en ocupaciones de adultos mayores con demencia, donde se hace referencia al término "psicología social maligna", el cual corresponde a un concepto desde la psicología, que se define como "el impacto negativo del ambiente psicosocial que altera la personalidad, la cual puede incluir traición, desempoderamiento, infantilización, intimidación, etiquetado, estigmatización e ignorancia" (Kitwood, 1997; Teitelman, Raber \& Watts, 2010, p. 323). A partir de dicho concepto, es posible visualizar que el grupo de adultos mayores que participan del taller de música se encuentran expuestos al estigma y prejuicios de los demás residentes que no participan del taller, pero sí los observan desde otros sitios. Además, debido a la estructura, las bajas posibilidades de modificaciones y adaptaciones durante las sesiones del taller observado, junto con la guía constante de desempeño por parte de los monitores hacia los residentes, lo cual dificulta las opciones para la espontaneidad de ellos, genera un contexto de dificultades para el empoderamiento de la muestra del estudio, siendo complejo para ellos poder conectarse o vincularse emocionalmente con las sesiones.

El ítem Realizar una actividad hasta lograrla/ completarla, independiente del éxito o fracaso, denota que la persona sentía la curiosidad e interés suficiente como para llevar la actividad hasta el final, no sólo por el resultado final de la actividad, sino que además por la propia percepción de sí mismo al llegar al final de dicha actividad (De las Heras et al, 1998).

El taller presentaba una estructura poco flexible y modificable por los usuarios. Los límites de tiempo, materiales y actividades previamente determinados, no son permeables, por lo que se observa una disminución por el interés de participary así, en consecuencia, existen dificultades para poder continuar con las actividades hasta completarlas o lograrlas. Se observa que aquellos participantes que requerían un tiempo mayor para el desempeño, no lograban completar la actividades, viéndose restringida su participación en la globalidad del taller.

\section{DisCUSıÓn}

En relación al ambiente y el impacto de éste sobre la expresión de la volición de los usuarios observados, se obtienen variaciones según momentos del taller, influyendo las características personales y ambientales de cada usuario, las cuales en conjunto generaron diferencias en cuanto a la forma en que cada residente expresaba su volición durante su participación en el taller, coincidiendo manifestar variaciones y formas similares durante algunos momentos claves de éste, como eran los espacios del inicio y término de la actividad con las melodías judías, o cuando tocaban instrumentos o cantaron canciones que pudieron reconocer. La forma en la cual expresan la volición corresponde a un nivel gestual y corporal, manifestando cambios posturales y faciales, siendo escasa la expresión verbal de ideas o comentarios volitivos. La expresión volitiva de la muestra tiene relación con los resultados obtenidos por el estudio de Raber et al (2010), donde se encuentra que los adultos mayores, por lo general, presentan una particularidad en su expresión volitiva, donde ésta ocurre mayormente a nivel gestual y corporal, pero a nivel verbal en diversas ocasiones no hay un mayor desarrollo, lo que no se condice con el componente no-verbal. Asimismo, en el presente estudio se les preguntó a los participantes correspondientes a la muestra su nivel de interés y motivación por participar en el taller, considerando además que cada uno asiste en forma voluntaria: el $100 \%$ expresó verbalmente sentir un alto nivel de interés por participar.

Por otro lado, se obtiene que el ambiente social, considerado dentro de las formas ocupacionales, genera un impacto e influencia sobre la expresión de la volición, lo cual se observa como un elemento que dificulta la expresión de dicha volición. Éste, en conjunto con el desarrollo de las actividades en sí, se presenta como un aspecto altamente estructurado, guiando paso a paso la participación de los residentes, viéndose limitada la espontaneidad y libre ejecución de acciones incluidas en la volición de las personas. Asimismo se comenta en el estudio de Raber et al (2010) que el ambiente 
social influye sobre la expresión de la volición de los usuarios, donde las personas alrededor de ellos son los que determinan finalmente el grado de motivación o interés por alguna actividad u ocupación, así es como el ambiente social es el que ejerce una influencia sobre las preferencias del usuario y el compromiso significativo con la actividad. Se establece que cuando los intereses o la expresión de intereses del usuario es reconocida por el resto (persona, cuidadores, monitores), éste aumenta sus deseos de participar en diferentes ocupaciones, aumenta su compromiso e involucramiento en actividades y además logra expresar mayor placer y comodidad con su desempeño en forma verbal y no verbal; en cambio, cuando la expresión del comportamiento volitivo no logra ser comprendido por los demás, el usuario disminuye y cesa su desempeño, aumenta la resistencia a la interacción, la frustración y ansiedad.

De este modo, se hace posible comprender las dificultades para observar la expresión de la volición de los adultos mayores dentro del taller, en donde se evidencia un ambiente con un nivel de influencia tal, que en interrelación con las características propias del cuadro de base de cada residente, se produce un impacto a nivel volitivo y en su expresión durante el desempeño de una actividad de interés.

Dentro del estudio, al presentarse un ambiente estructurado para el desempeño del taller, se dificulta el reconocimiento de la expresión del comportamiento volitivo por parte de los adultos mayores, por lo tanto, para los monitores y personal a cargo, se les torna más difícil poder determinar el placer o displacer al participar dentro del taller en general o en alguna de sus actividades dentro de la sesión. Así es como se dificulta la adaptación o modificación de las actividades o tareas durante las sesiones, que se adapten a las preferencias y habilidades del grupo, a partir de lo que podrían expresar tras cada sesión. Pero al existir una estructura con bajas posibilidades de adaptación y flexibilidad, se dificulta la expresión volitiva espontánea durante el desempeño personal del grupo de adultos mayores en las diferentes actividades correspondientes a sus intereses o inclinaciones.

Como Terapeutas Ocupacionales, esta investigación nos invita a mirar, no sólo en el individuo, en relación a sus características y condiciones personales, que los diferencia unos de otros para analizar su desempeño, si no que además nos invita a observar factores que, en variadas oportunidades, se encuentra fuera de la capacidad de control de los sujetos, produciendo una influencia positiva o negativa sobre éste. Por lo tanto surge la necesidad de formular estrategias para adaptar o modificar la forma en que la persona se desempeña, con sus propias características, dentro de dicho ambiente, buscando en todo momento potenciar o mejorar su desempeño y participación en actividades y ocupaciones. Es aquí donde el ambiente cobra importancia desde el impacto que ejerce sobre el desempeño de las personas en sus ocupaciones.

Finalmente, los resultados obtenidos y su posterior análisis permiten reflejar que es necesaria la generación de futuras investigaciones que profundicen sobre el componente volitivo en interacción con la música, en población mayor con deterioro cognitivo o demencia y el impacto ambiental en diferentes ambientes ocupacionales. 


\section{REFERENCIAS BibLIOGRÁFICAS}

Asociación Americana de Psiquiatría (2014). Guía de Consulta de los Criterios Diagnósticos del DSM-5. Arlington, Estados Unidos: Autor. American Occupational Therapy Association (2015). Dementia and the role ofOccupational Therapy. Recuperado de: http://www.aota.org/ about-occupational-therapy/professionals/mh/dementia.aspx Ayala, J. (2010). Fundamentos teóricos de la terapia ocupacional: La ocupación en la vejez. En Corregidor, I.A. (Ed). Terapia ocupacional en geriatría y gerontología: Bases conceptuales y aplicaciones prácticas (pp. 11-18). Madrid, España: SEGG.

Baranda, B. (2014). Prólogo. En Servicio Nacional del Adulto Mayor (senama) (Ed.), Chile y sus Mayores: Resultados Tercera Encuesta Nacional, Calidad de Vida en la Vejez (p. 6). Santiago, Chile. Recuperado de: http://www.senama.cl/filesapp/Chile\%20y\%20 sus\%20mayores\%202013,\%20Encuesta\%20de\%20Calidad\%20 de\%20Vida.pdf

De las Heras, C.G., Llerena, V., Kielhofner, G. (2003). Proceso de remotivación: Intervención progresiva para individuos con desafios volicionales severos. University of Illinois, Chicago. Estados Unidos: The Model of Human Occupation Clearinghouse.

De las Heras, C.G., Geist, R., Kielhofner, G., Li, Y. (1998). Manual del Usuario del Cuestionario Volicional. University of Illinois, Chicago. Estados Unidos: The Model of Human Occupation Clearinghouse.

Folstein, M.F., Folstein, S.E., McHugh, P.R., Fanjiang, G. (1975). “MiniMental State" a practical method for grading the cognitive state of patients for the clinician. Journal of Psychiatric Researches, 12, pp. 189-198. Recuperado de: http://turkpsikiyatri.org/arsiv/ mmse_folstein_mchugh.pdf

Jacobsen, J-H., Stelzer, J., Fritz, T.H., Chételat, G., La Joie, R., Turner, T. (2015). Why Musical Memory Can Be Preserved In Advanced Alzheimer's Disease. Brain, a journal of Neurology, 138(8), 24382450. Doi: 10.1093/brain/aww135.

Kielhofner, G. (2011). Volición. En Kielhofner, G. (Ed.), Modelo de Ocupación Humana: teoría y aplicación (pp. 32-49). Chicago, Estados Unidos: Panamericana.

Kielhofner, G. (2011). El entorno y la ocupación humana. En Kielhofner, G. (Ed.), Modelo de Ocupación Humana: teoría y aplicación (pp. 85-99). Chicago, Estados Unidos: Panamericana.

Kitwood, T. (1997). Dementia reconsidered: The person comes first. Buckingham, UK/Bristol, PA: Open University Press.

Ministerio de Salud (MINSAL) (2015). Documento Preliminar para la Elaboración del Plan Nacional para las Demencias. Santiago, Chile. Recuperado de: http://web.minsal.cl/sites/default/files/files/ PLAN_DEMENCIA_final.pdf

Pfeiffer, E. (1974). A short portable mental status questionnaire for the assessment of organic brain deficit in elderly patients. Journal of American Geriatrics Society. 23(10), pp. 433-441. DOI: 10.1111/j.15325415.1975.tb00927.x
Raber, C., Teitelman, J., Watts, J., Kielhofner, G. (2010). A Phenomenological Study Of Volition In Everyday Occupations Of Older People With Dementia. British Journal of Occupational Therapy, 73(11), 498-506. Doi: 10.4276/030802210X12892992239116

Servicio Nacional del Adulto Mayor (SENAMA) (2013). Estudio de Actualización del Catastro de Establecimientos de Larga Estadía (elEAM) de las Regiones de Tarapacá, Antofagasta, Atacama, Coquimbo, Valparaíso, Libertador Bernardo O'Higgins, Maule, Biobio, Araucanía, Los Lagos, Aysén del General Carlos Ibáñez del Campo, Magallanes y Antártica Chilena, Los Ríos, Arica y Parinacota y Región Metropolitana: Informe Final. Santiago, Chile. Recuperado de: http://www.senama.cl/ filesapp/INFORME\%20FINAL\%20CATASTRO\%20ELEAM\%20 NACIONAL\%202012\%20REVISADO.pdf

Teitelman, J., Raber, C., Watts, J. (2012). The power fo the social environment in motivating persons with dementia to engage in occupation: Qualitative findings. Physical \& Occupational Therapy in Geriatrics, 28(4)321-333. Doi: 10.3109/02703181.2010.532582. 
